\title{
Instantons on D-branes
}

\section{Citation}

Vafa, Cumrun. 1996. "Instantons on D-Branes." Nuclear Physics B 463 (2-3): 435-42. https:// doi.org/10.1016/0550-3213(96)00075-2.

\section{Permanent link}

http://nrs.harvard.edu/urn-3:HUL.InstRepos:41385068

\section{Terms of Use}

This article was downloaded from Harvard University's DASH repository, and is made available under the terms and conditions applicable to Other Posted Material, as set forth at http:// nrs.harvard.edu/urn-3:HUL.InstRepos:dash.current.terms-of-use\#LAA

\section{Share Your Story}

The Harvard community has made this article openly available.

Please share how this access benefits you. Submit a story.

Accessibility 
HUTP-95/A049

hep-th/9512078

\title{
Instantons on D-branes
}

\author{
Cumrun Vafa \\ Lyman Laboratory of Physics, Harvard University \\ Cambridge, MA 02138, USA
}

\begin{abstract}
We consider type IIA compactification on $K 3$. We show that the instanton subsectors of the worldvolume of $N$ 4-branes wrapped around $K 3$ lead to a Hagedorn density of BPS states in accord with heterotic-type IIA duality in 6 dimensions. We also find evidence that the correct framework to understand the results of Nakajima on the appearance of affine Kac-Moody algebras on the cohomology of moduli space of instantons on ALE spaces is in the context of heterotic-type IIA string duality.
\end{abstract}

Dec. 1995 
One of the motivations behind the recent proposals of string dualities has been the appearance of field theory dualities, and in particular the Montonen-Olive duality for $\mathrm{N}=4$ YM theory. The existence of field theory dualities is a necessary condition to any generalization to string theory. There seems to be, however, another indirect, but perhaps deeper connection between the string duality and $N=4 \mathrm{YM}$ duality. In [1] a strong coupling test of Montonen-Olive S-duality was done. In particular $S U(2), N=4 \mathrm{YM}$ on $K 3$ was considered and it was found that the partition function of the theory is modular, in accord with S-duality. However, there was a surprising aspect to this computation: In the course of this computation the oscillator partition function of bosonic string theory made an unexpected appearance! Technically this arose by relating the moduli space of $S U(2)$ instantons on $K 3$ to the moduli space of some number of points on $K 3$. A similar coincidence that was noticed was that the partition function of $n$ unordered points on $T^{4}$ similarly gives the oscillator partition function for superstrings. It was suggested in [2] that these coincidences are not accidental and related to string dualities: type II-type II duality (on $T^{4}$ ) or type IIA (on $K 3$ )-heterotic (on $T^{4}$ ) duality. In particular it was shown there that a gas of 0 -branes on $T^{4}$ in the presence of one 4-brane wrapped around $T^{4}$ form bound states in accordance with predictions from string duality, where the relevant moduli space that arises is that of a number of points on $T^{4}$. This generalized the result of [3] [4] who showed (the $T$-dual of the statement) that one 0 -brane and one 4-brane form a bound state with the expected degeneracy. For heterotic-type II duality, the suggestion of [2] was realized in [5] where it was shown that again the relevant moduli problem that arises, whether it be for a gas of 0-branes in the presence of a 4-brane, or a 2-brane wrapped around a 2-cycle of $K 3$, is that of a symmetric product of a number of $K 3$ 's.

Even though these results are a strong confirmation both of the string dualities and the D-branes as the relevant solitons [6] (see also the related work [7] [2] [3] [4] [3] [5] [9] [10] [11] [12] [13] [14] [15] [16]) two issues remained, one real and one aesthetic: The first one was what happens if we consider other configurations, for example what if we have $N$ wrapped 4-branes instead of one? Of course by T-duality on $K 3$, one could map the problem to the cases already considered, but one would also like to be able to tackle this problem directly. It is quite interesting that the flavor of the field theory questions changes so dramatically once one uses $T$-duality. This suggests that $T$-duality can be used to learn about non-trivial field theory properties, such as strong/weak duality in N=4 YM. Another issue mainly aesthetic, is that it seemed that we had stumbled upon bosonic string partition function in 
[1] quite accidentally. It would have been much more satisfactory if not only the mathematical structure but also the physics of the moduli problem encountered be the same for testing of both the string duality and the Montonen-Olive duality. We shall argue in this paper that these two issues nicely complement one another and show that by considering the configuration of $N$ 4-branes wrapped around $K 3$ the physics of the counting of the Hagedorn density of BPS states will coincide with the computation done in [1] and so that computation can also be reinterpreted as a test of type II-heterotic string duality! That the physics may be identical is not surprising when we recall that $N$ wrapped 4 -branes around $K 3$ will give rise to $U(N)$ gauge theory on $K 3 \times \mathbf{R}$ where $\mathbf{R}$ plays the role of time. In fact the number of supersymmetries is such that the static configurations of the 4-brane is identical to $N=4 \mathrm{YM}$ on $K 3$.

Our starting point is the observation [7] that since the dynamics of $\mathrm{N}$ D-branes is captured by the maximally supersymmetric $U(N)$ gauge theory on the worldvolume of the D-brane, the normalizable ground states of this gauge theory can be reinterpreted as bound states of $N$ D-branes. We will need to generalize this observation for our purposes: What is the interpretation of excited states of this supersymmetric $U(N)$ gauge theory on the worldvolume of the D-brane? Clearly they should be identified with the excitation of the bound states. These excitations are generically unstable. However, let us consider a BPS state of the $U(N)$ gauge theory. This in particular means that it is a reduced supersymmetry multiplet of the $U(N)$ theory. Since we can think of the supersymmetry of the $U(N)$ theory as coming from the 'pull back' of the supersymmetries in spacetime in the presence of D-brane bound state, we can interpret these states as bound states which correspond to excitations of D-brane bound states which are BPS saturated and thus stable 1. Clearly the size of the spacetime supersymmetry multiplet the bound state will reside in will depend on how many supersymmetries the corresponding BPS state preserves in the $U(N)$ worldvolume theory.

If we have $N 4$-branes wrapped around the $K 3$, we are dealing with a 5 dimensional $U(N)$ gauge theory on $K 3 \times \mathbf{R}$ where $\mathbf{R}$ is the time direction. Thus the instantons on $K 3$, which preserve half the supersymmetries of the theory, can be viewed as the BPS states of

1 A good analogy to keep in mind is the case of 1-branes, were the BPS states of the worldsheet theory, in the Green-Schwarz formulation, correspond to excited states of the string which are killed by half of the supersymmetries on the worldsheet (say the left-moving supersymmetries). Clearly these correspond also to BPS states in the spacetime [17]. In the above we are generalizing this observation to Dirichlet p-branes. 
this theory. Consider the instanton number $k$ sector of this theory, which should be viewed as a particular Hilbert space subsector of this 5-dimensional theory. The situation at hand is very similar to that considered in [18]. There are two possibilities that may arise: For generic point on moduli of instantons the gauge symmetry is completely broken and we can integrate out the gauge field also in the $\mathbf{R}$ direction. If this were the full story we would obtain as the effective theory on $\mathbf{R}$ the sigma model on instanton moduli space and the ground states would be identified with the cohomology elements of instanton moduli space. However it may happen that for some singular loci of moduli space of instantons we have (partially) enhanced gauge symmetry which would imply that we cannot ignore the dynamics of the gauge theory on $\mathbf{R}$, and in particular there will be a mixture of guage theory coupled in a non-trivial way to a sigma model (on the rest of the instanton moduli space). This would be very complicated to deal with. In fact whether one is in the 'good case' or 'bad case' should be reflected by the nature of the singularities of the instanton moduli space. In particular the 'bad case' should be accompanied with singular instanton moduli space, for which one does not have a canonical recipe for repairing the singularity and that the left over gauge dynamics on $\mathbf{R}$ would be the physical information needed for the resolution of the singularity. This situation is indeed similar to what was encountered in [18] were in the 'good case' there was a smooth moduli space (the Hitchin space) and in the bad case there were singularities with no canonical resolution. The nature of singularities of instanton moduli space is thus a strong hint whether one will have to include extra dynamics in addition to the sigma model on the instanton moduli space.

Let us denote the moduli space of $S U(N)$ instantons on $K 3$ with instanton number $k$ by $\mathcal{M}_{k}^{N}$. The dimension of $\mathcal{M}_{k}^{N}$ is

$$
\operatorname{dim}\left(\mathcal{M}_{k}\right)=4[N(k-N)+1]
$$

Let us recall that $N$ D-branes will have a $U(N)$ gauge symmetry on their worldvolume [19] 20], where the $U(1) \subset U(N)$ is identified with the center of mass degree of freedom of the D-brane [7]. Since for a generic metric on $K 3$ there are no $U(1)$ instantons we can ignore the $U(1)$ dynamics, and simply concentrate on the $S U(N)$ part 2 .

2 Since $U(N)$ is not quite the direct product of $U(1)$ and $S U(N)$ in general this means that there are 't Hooft electric and magnetic flux allowed on the manifold correlated with the $U(1)$ electric and magnetic flux. This in fact was a key point in the considerations of [7]. Here, since the $U(1)$ field is trivial we do not have any 't Hooft $Z_{N}$ fluxes turned on. 
Before getting into the detail of the analysis we would like to know what charges the BPS states would carry, in order to compare with the expected degeneracy of states based on string duality. In particular we would like to compute $P^{2} / 2$ where $P^{2}=P_{R}^{2}-P_{L}^{2}$ on the heterotic side is identified with the length squared of the momentum vector and $P_{R}, P_{L}$ are right and left momenta of a vector in the Narain lattice $\Gamma^{4,20}$. The degeneracy of the BPS state (apart from the supersymmetry multiplet degeneracy) is expected to be $d\left(\frac{P^{2}}{2}+1\right)$ where $d(n)$ denotes the degeneracy of $n$-th oscillator level of left-movers of bosonic strings, i.e.

$$
\eta(q)^{-24}=q^{-1} \sum d(n) q^{n}
$$

where $\eta$ is the Dedekind eta function. By identifying the $\Gamma^{4,20}$ with the integral cohomology of $K 3$, we see that a $\Gamma^{1,1}$ part of it will correspond to 0 - and 4-dimensional cohomologies. This in particular means that if we have $N$ 4-branes and $M 0$-branes, we would identify the $P^{2} / 2$ with $N M$. To begin with we have $N 4$-branes and no 0 -branes, so one may think we are in the situation corresponding to $P^{2}=0$. This is not the case. It has been pointed out in [5] that in general there are quantum corrections which lead to anomalous charges. In particular it was argued there that a 4-brane on $K 3$ will acquire in addition a -10 -brane charge. This arose because on the worldvolume there is a correction on the worldvolume of the 4-brane (converting from type IIB considered in [5] to type IIA)

$$
\frac{1}{48} \int A \wedge p_{1}(R)
$$

where $A$ is the 1 -form RR field coupling to the 0 -branes and $p_{1}(R)$ is the first Pontryagin class made of the Riemann tensor. For $K 3$ this is $p_{1}=-48$ and so we get a net effect on $\mathbf{R}$ of integration $-\int A$, i.e. we have -1 unit of 0 -brane charge. For $N$ 4-branes that we are considering we would thus get, in addition to the 4-brane charge, $-N$ units of 0 -brane charge. Since we are considering instanton number $k$ of $S U(N)$ we should ask whether or not there are any other relevant corrections which would lead to a different counting of the 0-branes. Indeed there is. The argument outlined in [5] actually predicts in addition a term of the form

$$
\int A \wedge c_{2}(F)
$$

where $c_{2}(F)$ is the second Chern class of the $S U(N)$ gauge field living on the 4-brane worldvolume. In fact, as was pointed out to us by Douglas, this term is indeed generated [21] (see also [22] [10]). Noting that $c_{2}(F)=k$ is the instanton number of the $S U(N)$ 
gauge group, we learn that in the instanton number $k$ sector of the $S U(N)$ gauge theory we have in addition $k$ units of the 0-brane charge. Thus the total effective 0-brane charge $M$, taking into account the above corrections 3 , is $M=k-N$. We thus have

$$
\frac{1}{2} P^{2}=N M=N(k-N)
$$

Before getting into the analysis of the moduli space of instantons, we can anticipate the 'good' and 'bad' cases based on what we have just found. One sign of the 'bad' cases would be finding no mass gap. This will be the case if $N$ and $k$ are not relatively prime. To see this note that if $N$ and $k$ are not relatively prime then $N$ and $M=k-N$ are also not relatively prime, which implies that the BPS state we are about to find does not correspond to a primitive charge in the $\Gamma^{20,4}$ lattice. Thus it has the same energy as two subsystems. Since these subsystems can be separated to arbitrary distances we thus have no mass gap and we would be up against finding a bound state at threshold. This lack of mass gap should be reflected in the severe singularities of moduli space of $S U(N)$ instantons with instanton number $k$ not relatively prime to $N$. On the other hand if $k$ and $N$ are relatively prime, we should expect a mass gap and so the corresponding moduli space of instantons has to have a discrete spectrum near the origin. This suggests that in such cases the instanton moduli space should be smooth enough to allow this. So we have tentatively identified the 'good' and 'bad' cases by checking whether $k$ and $N$ are relatively prime (good) or not (bad). Below we will find some indications which go in the direction of confirming this picture.

The moduli space of instantons on $K 3$ is most intensively studied for $S U(2)$ (see [1] for the relevant references). From the above discussion we thus expect that in this case for $k$ odd we get a good moduli space. This is indeed the case and we find that the cohomology of $\mathcal{M}_{k}^{2}$ for $k$ odd is the same as the cohomology of $2 k-3$ unordered points on $K 3$, and moreover for some choice of complex structure on $K 3$ the moduli space itself coincides with

$$
\mathcal{M}_{k}^{2}=(K 3)^{2 k-3} / S_{2 k-3}
$$

The Euler character of this space, as noted in $\mathbb{1}$ is $d(2 k-3)$ (where $d(n)$ was defined in (2)). From string duality we should have expected

$$
d\left(\frac{1}{2} P^{2}+1\right)=d(2(k-2)+1)=d(2 k-3)
$$

3 The relative sign contributions has not been checked but, just as in [23], it should be so in order to be consistent with string duality 
which is thus a confirmation of the prediction of heterotic-type II string duality. For $k$ even, the above discussion suggests that the moduli space should be badly singular and the extra gauge dynamics will have to be taken into account for its resolution. That the space is singular is well known mathematically. In fact this was one of the difficulties in fully checking the prediction of Montonen-Olive duality on $K 3$ studied in [1]. The $N=4$ duality in this case predicts a form for the Euler characteristic of all the even cases as well, but the number is fractional [1], again indicating that the moduli space is singular. A fractional number cannot be counting the number of bound states. In fact as discussed above we expect the string theory computation of bound states at threshold to differ from the computation of the Euler characteristic of this space.

How about for other values of $N>2$ ? Unfortunately this space is not well studied mathematically. But it is natural to conjecture the following (which was also conjectured in [1] based on $N=4$ duality): The space $\mathcal{M}_{k}^{N}$ for $N$ and $k$ relatively prime is 'smooth' ( or more precisely has a canonical smooth resolution) and its cohomology is the same日 as that of $N(k-N)+1$ unordered points on $K 3$. First of all note that the dimension of the space of $N(k-N)+1$ points on $K 3$ agrees with the dimension of the moduli space $\mathcal{M}_{k}^{N}$ given in (1). Moreover, if this conjecture is true it gives the correct prediction for the degeneracy of the bound state, namely we expect to get $d(N M+1)=d(N(k-N)+1)$ as the degeneracy and this is exactly the cohomology of the space of $N(k-N)+1$ unordered points on $K 3$. The strongest evidence for this conjecture, apart from the fact that it would be a natural generalization from the $S U(2)$ case, is that it is in fact true at least for all instanton numbers $k=1 \bmod N$ [24].

Let us note very briefly the situation with type II-type II duality on $T^{4}$. In this case the dimension of the moduli of $U(N)$ instantons with instanton number $k$ is $4 k N+4$ (taking into account the $U(1)$ flat bundle on $T^{4}$ ). Moreover the effective 0 -brane charge is $k$ (as $p_{1}$ of $T^{4}$ is zero we do not get an additional $-N$ that we got for $K 3$ ). To be in accord with string duality (using the observation in [1] that the cohomology of $n$ unordered points on $T^{4}$ agrees with the superstring oscillator degeneracy at level $n$ ) it is natural to

4 Since by $T$-duality we can convert a 4 -brane to 0 -brane, i.e. a point on $K 3$, it seems that this mathemetical conjecture may have a simple physical interpretation. In particular the proof in the case of $S U(2)$ involves relating instanton moduli to points on $K 3$ which can be viewed as the zeros of a canonical zero mode of a field transforming in the fundamental of $U(2)$. Since for a 0-brane and a 4-brane a field in the fundamental naturally arises [8] this mathematical construction may have a natural physical interpretation. 
conjecture that if $k$ and $N$ are relatively prime the moduli space of $S U(N)$ instantons is (or more precisely has the same cohomology as) the $k N$ fold symmetric product of $T^{4}$, and together with the $U(1)$ flat moduli we would thus have the moduli space $T^{4} \times\left(T^{4}\right)^{k N} / S_{k N}$. It would be interesting to check this conjecture.

Let us close by making one remark in connection with the work of Nakajima [25] on instantons on ALE spaces. There has already been some discussion of this work in the physics literature [1] [26]. Nakajima shows that if we consider $U(N)$ guage group on an ALE space, say $A_{r-1}$ for concreteness, we get an action of $S U(r)$ Kac-Moody algebra at level $N$ acting on the cohomology of $U(N)$ instanton moduli space. In defining this action the vanishing 2-cycles of the ALE play a crucial role. It was suggested in [1] that a proper understanding of this may involve at least a 5-dimensional theory, where the instanton cohomologies would be viewed as BPS states and that string dualities may play a role. Here we would like to suggest that this is indeed the case where we identify the missing 5-dimensional theory with the worldvolume theory of the 4-brane. In fact based on string dualities [27] [28] [8] and more directly using the D-branes [5] one expects to have an enhanced gauge symmetry when a 2-cycle wraps around a vanishing $S^{2}$. The gauge particles are identified with 2-branes wrapped around the 2-cycles. For ALE corresponding to ADE we expect to get the corresponding ADE gauge symmetry. Now consider type IIA compactification on a singular $K 3$. In this case the relevant singularity can be modeled by an ALE space. Now, by heterotic-type II duality, we would land on a heterotic background near an enhanced gauge symmetry point. Moreover the instanton cohomologies will arise from oscillator modes of heterotic strings. Thus the action of the 2-branes on the instanton cohomology space can be reinterpreted on the heterotic side as the action of the affine KacMoody current algebra on the string oscillator states! Note that this is in accord with the fact that the currents on the heterotic side are left-movers and the BPS states all come from the left-movers tensored with the ground state of the right-movers. The puzzle at first sight may be that we expect that the heterotic side leads to a vertex operator algebra at level 1 and not $N$. There is a natural resolution of this puzzle: As discussed above, $N$ 4-branes will not give all the oscillator states, but all the oscillator levels separated by $N$ units $\left(N_{L}=N \cdot M+1\right.$ where $M$ varies). Is there any subalgebra of the Kac-Moody algebra which acts on this subspace? Indeed there is: Consider $J_{n}$ to be the current of the affine Kac-Moody algebra. Consider $\hat{J}_{n}=J_{n N}$. Since we have modified the moding by $N$ times the basic quantum of oscillation this subalgebra can now act on the cohomology of instanton moduli space of $S U(N)$ which have oscillation gaps of $N$ units. Moreover, it 
is an easy exercise to see that if the original affine Lie algebra has level 1, as is predicted by string duality, the $\hat{J}_{n}$ form an affine Lie algebra, but now at level $N$. We find this a strong hint that the natural interpretation of Nakajima's results is in the context of type II-heterotic string duality.

We would like to thank M. Bershadsky, M. Douglas, V. Sadov and S. Sethi for valuable discussions. The research of C. V. is supported in part by NSF grant PHY-92-18167. 


\section{References}

[1] C. Vafa and E. Witten, Nucl. Phys. B431 (1994) 3

[2] C. Vafa, Gas of D-Branes and Hagedorn Density of BPS States, HUTP-95/A042, hep-th/9511088

[3] A. Sen, A Note on Marginally Stable Bound States in Type II String Theory, MRI/PHY/23-95, hep-th/95102229

[4] A. Sen, U-duality and Intersecting D-branes, MRI/PHY/23-95, hep-th/9511026

[5] M. Bershadsky, V. Sadov and C. Vafa, D-Branes and Topological Field Theories hepth/9511222

[6] J. Polchinski, Dirichlet-Branes and Ramond-Ramond Charges, NSF-ITP-95-122, hepth/9510017

[7] E. Witten, Bound States Of Strings And p-Branes, hep-th/9510135

[8] M. Bershadsky, V. Sadov and C.Vafa, D-Strings on D-Manifolds, HUTP-95/A035, IASSNS-HEP-95-77, hep-th/9510225

[9] E. Witten, Small Instantons in String Theory, hep-th/9511030

[10] M. Li, Boundary States of D-Branes and Dy-Strings, hep-th/9510161

[11] C. Bachas, D-brane dynamics, hep-th/9511043

[12] C. G. Callan, and I. R. Klebanov, D-Brane Boundary State Dynamics, hep-th/9511173

[13] T. Banks and L. Susskind, Brane - Anti-Brane Forces, hep-th/9511194

[14] A. Strominger, Open P-Branes, hep-th/9512059

[15] P. Townsend, D-branes from M-branes, hep-th/9512062

[16] R.R. Khuri and R.C. Myers, Rusty Scatter Branes, hep-th/9512061

[17] A. Dabholkar and J. Harvey, Phys. Rev. Lett. 63 (1989) 478

[18] M. Bershadsky, A. Johansen, V. Sadov and C. Vafa, Nucl. Phys. B448 (1995) 166

[19] J. Dai, R. G. Leigh and J. Polchinski, Mod. Phys. lett, A4 (1989) 2073

[20] P. Horava, Phys. Lett. B231 (1989) 251

[21] M. Douglas, Branes within Branes, hep-th/9512077

[22] C.G. Callan, C. Lovelace, C.R. Nappi and S.A. Yost, Nucl. Phys. B308 (1988) 221

[23] C. Vafa and E. Witten, Nucl. Phys. B447 (1995) 261-270

[24] P. Kronheimer, private communication.

[25] H. Nakajima, Homology of moduli spaces of instantons on ALE Spaces. I, J. Diff. Geom. 40(1990) 105; Instantons on ALE spaces, quiver varieties, and Kac-Moody algebras, preprint; Gauge theory on resolutions of simple singularities and affine Lie algebras, preprint.

[26] A. Losev, G. Moore, N. Nekrasov and S. Shatashvili, Four-Dimensional Avatars of Two-dimensional RCFT, Talks given at Strings 95 and ICTP Conf. on S Duality and Mirror Symmetry, hep-th/9509151

[27] C. Hull and P. Townsend, Nucl. Phys. B360 (1995) 109.

[28] E. Witten, Nucl. Phys. B443 (1995) 85 